\title{
Dynamical Stripe Correlations in Cuprate Superconductors.
}

\author{
J. Zaanen, O. Y. Osman, H. Eskes and W. van Saarloos \\ Lorentz Institute for Theoretical Physics, Leiden University, P.O.B. 9506, \\ 2300 RA Leiden, The Netherlands
}

Based on the recent observation of the stripe instability in Cuprate superconductors, we present the hypothesis that the normal state finds its origin in a particular kind of stripe-quantum fluid. The charged domain walls are interpreted as strings on a lattice and the quantum fluctuation of an individual string is driven by a proliferation of kinks. The kink dynamics gives rise to meandering fluctuations of the string as a whole. We identify a special string vacuum characterized by a proliferation of charged kinks. This state carries a Luttinger-liquid like electronic excitation spectrum. PACS numbers: $64.60 .-i, 71.27 .+a, 74.72 .-h, 75.10 .-b$

\section{INTRODUCTION}

There is a widespread belief that the electron-fluid realized in cuprate superconductors is unrelated to the normal metallic state described by Fermi-liquid theory. Emery and Kivelson were the first to point out the possibility that even the quasiparticle concept itself could be irrelevant in cuprates. ${ }^{1}$ They suggested the possibility of dynamical phase separation: the carriers would segegrate in regions which would persist as fluctuating quantities in the metal and the superconductor.

Nature seems to have found an even more attractive solution. The carriers form line-like many particle bound states which are at the same time anti-phase boundaries in the Néel spin background (charged domain walls). It was found some time ago that these textures correspond with the ground states of semiclassical mean field theory in doped Mott-Hubbard insulators. ${ }^{2}$ Subsequently, ordered charged domain wall structures were found experi- 
mentally in both two dimensional nickelates and manganites, which appear to be electronically more strongly localized than the cuprates. ${ }^{3}$ Initially, these stripes were considered as rather far-fetched in the context of high $T_{c}$ superconductivity, but this changed radically with the experimental discovery by Tranquada et al. ${ }^{4}$ that these stripes actually freeze out at a doping concentration $x \simeq 1 / 8$ in a system showing the so-called LTT (low temperature tetragonal) lattice deformation.

It was a long standing mystery why at doping concentrations $x=1 / 8$ the superconductivity vanished in systems showing the LTT deformation ( $\mathrm{La}_{2-x} \mathrm{Ba}_{x} \mathrm{CuO} \mathrm{O}_{4}$ and $\mathrm{La}_{2-x-y} \mathrm{Nd}_{y} \mathrm{Sr}_{x} \mathrm{CuO} \mathrm{O}_{4}$ ). Using neutron scattering, Tranquada et al showed that a static striped phase appears, and because the holes are bound to the stripes this state is electrically insulating. A strong case can be made that stripe correlations will persist in the metallic. state. ${ }^{4}$ The arguments are straightforward: (i) the LTT deformation acts as a collective pinning potential which is only effective if the fluid is stripe-like, (ii) at least the spatial aspects of the dynamical incommensurate spin fluctuations seen in the metallic and superconducting states fit the expectations for a striped fluid.

We have focussed our attention on the question in how far the experimental reality in the cuprates can be recovered from the 'stripe-only' limit. We consider the case where the bare holes are tightly bound into charged domain walls. ${ }^{5}$ The physics at low energy is then governed by the collective quantum- and thermal fluctuations of the domain walls themselves, in addition to the degrees of freedom of the spins inside the magnetic domains. Specifically, in the light of the experience with incommensurate (domain wall) fluids, the most important stripe degree of freedom should be its meandering fluctuation. We showed elsewhere that the peculiar dynamics of the spin fluctuations observed in the normal state appears quite naturally in the context of the thermal meandering fluctuations in a stripe (incommensurate) fluid. ${ }^{6}$ At the same time, it is fact that the vacuum in the high- $T_{c}$ superconductors carries also excitations which mimick fermion-like quasiparticles and at first sight it is much less obvious what these fermions have to do with fluctuating stripes. Here we will summarize our work on a class of models ('quantum lattice strings') ${ }^{7}$ which might describe the short time dynamics of the stripes. The collective motions of these strings are driven by a microscopic kink dynamics and there are distinct phases where kinks proliferate which carry half a hole. ${ }^{8}$ These kinks seem to form Luttinger liquids and the external electron or hole attaches to these kinks in a way which is similar to what is found in 1+1D electron systems. The single electron spectral function shows distinct cusps which at first sight look like quasiparticle peaks, spanning up a big fermi-surface already at low kink concentration. 


\section{Quantum lattice strings}

In addition to the 'stripe only' hypothesis, we assume that the microscopic dynamics of the charged domain walls is dominated by commensuration effects: (i) a lattice commensuration: the holes, and thereby the stripes, tend to localize on lattice sites. (ii) An intra-stripe charge commensuration: special stability is obtained when every hole adds a definite unit of length to the stripe, and this length may depend on the orientation of the stripe. Specifically, we assume that the maximum length added by one hole cannot exceed $2 a$, where the link to the next hole is oriented along the $(1,0) /(0,1)$ direction (Fig. 1). This corresponds with the charge commensuration observed in the ordered cuprate striped phase. In addition, one hole adds only a length $\sqrt{2} a$ when the stripe is oriented along a $(1,1)$ and equivalent directions, as is the case in the nickelates. As a consequence, adding a physical hole to a $(1,0)$ stripe causes a double kink (Fig. 1). By single hole hoppings, this double kink (and thereby the hole) delocalizes into a pair of propagating kinks. If these kinks proliferate, the stripe as a whole might delocalize.

\begin{tabular}{|c|c|}
\hline 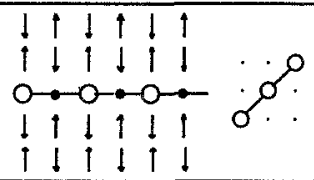 & 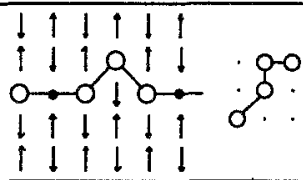 \\
\hline
\end{tabular}

Fig. 1. charge domain wall in the cuprate (left) and the $(1, \pm 1)$ kinks assumed to be responsible for the quantum melting of the striped phase. Notice that the on-stripe doubling of the unit cell is not seen experimentally, and is only indicated for counting purposes. The corresponding quantum lattice string configurations are also indicated

The above can be further abstracted by postulating quantum lattice string models. ${ }^{7}$ These models describe a collection of $N$ 'holes' forming all possible connected one dimensional trajectories on a two dimensional square lattice. The connectedness is defined by local rules. The minimal string is obtained by insisting that the links between the holes connect either nearestneighbour $((0,1),(1,0)$, 'horizontal') or next nearest neighbour $((1,1)$, etc., 'diagonal') sites on the square lattice (Fig. 2b). The $(1,1)$ links are the maximal length connections in the model, and correspond with the $(1,0)$ stripes in the cuprates, while the $(1,0)$ links in the model are equivalent to the $(1,1)$ kinks in the cuprates. Let $\left(\eta_{l}^{x}, \eta_{l}^{y}\right)$ be the position of hole $l$. We write the classical potential energy as,

$$
\mathcal{H}^{C l}=\sum\left[\mathcal{K} \delta\left(\left|\eta_{l+1}^{x}-\eta_{l}^{x}\right|-1\right) \delta\left(\left|\eta_{l+1}^{y}-\eta_{l}^{y}\right|-1\right)\right.
$$




$$
\left.+\sum_{i, j=0}^{2} \mathcal{L}_{i j} \delta\left(\left|\eta_{l+2}^{x}-\eta_{l}^{x}\right|-i\right) \delta\left(\left|\eta_{l+2}^{y}-\eta_{l}^{y}\right|-j\right)\right]
$$

The single-link term $(K)$ represent the energy differences between $(1,0)$ and $(1,1)$ links, while the two-link term $\left(L_{i j}\right)$ represents discretized curvature energy. The energies of various string configurations are indicated in Fig. $2 \mathrm{a}$. The string is quantized by introducing conjugate momenta $\hat{\pi}_{l}^{\alpha},\left[\hat{\eta}_{l}^{\alpha}, \hat{\pi}_{l^{\prime}}^{\alpha^{\prime}}\right]=$ $i \delta_{l, l^{\prime}} \delta_{\alpha, \alpha^{\prime}}$. A term $e^{i \lambda \hat{\pi}_{l}^{\alpha}}$ causes hole $l$ to hop a distance $\lambda$ in the $\alpha$ direction. The simplest, nearest neighbouring form for the kinetic energy is,

$$
\mathcal{H}_{Q u}=\mathcal{J} \sum_{l} \mathcal{P}_{S t r}^{x}(l) \mathcal{P}_{S t r}^{y}(l)\left(e^{i \hat{\pi}_{l}^{x}}+e^{-i \hat{\pi}_{l}^{x}}+e^{i \hat{\pi}_{l}^{y}}+e^{-i \hat{\pi}_{l}^{y}}\right)
$$

where $\mathcal{P}_{S t r}^{\alpha}(l)$ is a projector restricting the motion of hole $l$ to string configurations.

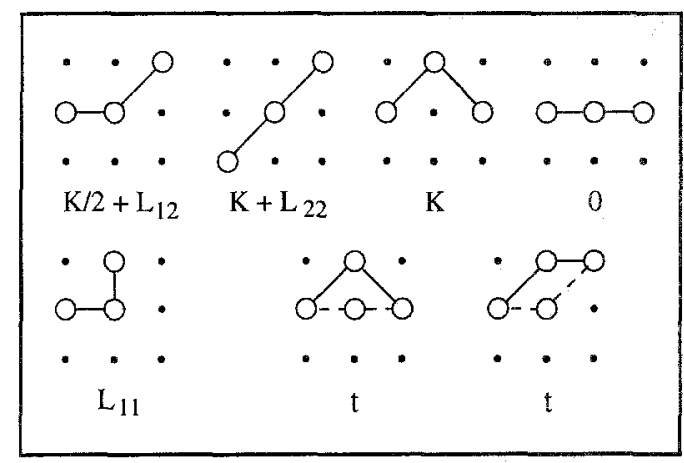

Fig. 2. Energies and tunneling amplitudes of the various local configurations of the strings.

From a theoretical point of view, the above model is rather interesting: it describes a $1+1 \mathrm{D}$ dynamics, subject to $2+1 \mathrm{D}$ boundary conditions via the geometric interpretation of the dynamics in terms of strings. The latter 'embedding' problem renders these string problems to be more rich than mere one dimensional problems. To identify the nature of the underlying $1+1 \mathrm{D}$ problem it is useful to neglect the boundary conditions altogether. Consider an infinitely long string and single out one point, the 'guider point'. The motion of this single point becomes irrelevant in the thermodynamic limit and one can now consider the problem entirely in terms of the link variables $\left(\delta \eta_{l}^{x}, \delta \eta_{l}^{y}\right)$ with $\delta \eta_{l}^{\alpha}=\eta_{l+1}^{\alpha}-\eta_{l}^{\alpha}$. Hence, this is like a one-dimensional lattice with a pair of dynamical variables on every site, taking both the values $\delta \eta^{\alpha}=0, \pm 1$. Associating these dynamical variables with the $M_{s}$ states of 
the local spin, the dynamical problem maps onto a system of two locally coupled $S=1$ quantum spin chains. ${ }^{7,8}$

Alternatively, a quantum string should correspond with a surface ('world sheet') in Euclidean space time. By means of the Suzuki-Trotter mapping one finds that the present string maps onto the problem of two coupled restricted solid-on-solid (RSOS) models, where the two height flavors correspond to $\eta_{l}^{x}$ and $\eta_{l}^{y}$. In fact, this equivalence between $1+1 \mathrm{D}$ quantum spin problems and quantum strings was implicitely exploited in the seminal work by den Nijs and Rommelse, ${ }^{9}$ dealing with the $S=1$ quantum Heisenberg chain. Let the string step forwards always in for instance the $x$ direction $\left(\delta \eta_{l}^{x}=1, \forall l\right)$ : the 'directed' string. In this case, a single spin chain remains, corresponding with the motions of the string in the $y$ direction. At the Heisenberg point of the spin problem, a partial ordering occurs on the string: the string as a whole is localized in the horizontal $(1,0)$ direction, but diagonal kinks proliferate which loose their positional order, but keep their alternating order: on every $(1,1)$ kink follows a $(1,-1)$ kink and vice versa, although the number of $(1,0)$ (horizontal) links between these kinks is arbitrary. This topological order explains the incompressible nature of the fluid realized in the spin chain.

The directed strings are characterized by an average direction in space. This is unrelated to a symmetry of the model Eq.'s $(1,2)$. Our finite size studies suggest that the strings commonly undergo a zero temperature ${ }^{11}$ spontaneous symmetry breaking to a directed state. Extreme curvature is needed for the string to loose its direction and this costs both kineticand potential energy. We studied systematically the phase diagram of the directed string/spin-chain/RSOS surface system. ${ }^{7}$ In addition to the six known phases, ${ }^{9}$ we discovered 4 new phases. Altogether, there are five classical phases (e.g. flat strings along $(1,0)$ or $(1,1)$ directions), three partial ordered phases (like the Heisenberg spin chain) and two quantum delocalized phases which are both of the free string variety, showing a meandering length increasing as the logarithm of the arclength.

\section{The fermionic excitations.}

In the above, an essential aspect is neglected altogether: the embedding of the string in $2+1 \mathrm{D}$, and its interpretation as bound state of holes. It is natural to consider open spatial boundary conditions, and the allowed string configurations are those where both the begin- and end point of the string lie on the surface. Consider a $L \times L$ square. The longest distance in this lattice corresponds with the line connecting two opposite corners, $L_{\text {max }}=\sqrt{2} L$. Given $\mathrm{N}$ holes, the longest ('fully stretched') string has length $\sqrt{2} N$ and 
when $N=L$ the string can just span the largest distance in the lattice. As a consequence, by local moves (hops of the holes) the string can deform to connect any pair of boundaries of the lattice (figure 3). For directed strings, the string boundary conditions are automatically taken into account, and we call this the 'saturated string'. Alternatively, it is also possible to 'oversaturate' the string by adding more holes than necessary to cover the diagonal. This excess length gives rise to excess curvature, and it is convenient to define a 'characteristic' configuration where this curvature is stored in pairs of $(1,0)-(0,1)$ kinks with regard to an otherwise fully stretched string (Fig. 3). ${ }^{10}$ Although this additional curvature is energetically unfavourable; it is controlled by an independent parameter: the thermodynamic potential of the holes. As function of this extra parameter, we find a number of additional phases which carry fermion-like excitations.

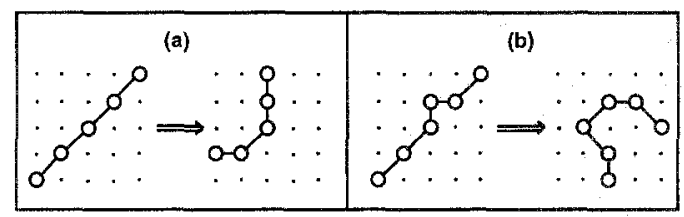

Fig. 3. The saturated string on a $5 \times 5$ lattice (a) and the string oversaturated with one hole (b). The characteristic configurations are indicated, as well as configurations which can be reached by a sequence of single-hole hopping processes.

It is interesting to consider the single-electron spectral function. In (inverse) photoemission, length is added (removed) from the strings and it is natural to assume that the hole is attached (removed) from the string in a local process. Specifically, every string configuration $\{\eta\}$ is written as a $N$ spinless fermion state $|\{\eta\}\rangle=\prod_{l=1}^{N} a_{\vec{\eta}_{l}}^{\dagger}|v a c\rangle$ and the string vacuum is $\left|\Phi_{0}\right\rangle=\sum_{\{\eta\}} \alpha(\{\eta\})|\{\eta\}\rangle$. The external electron $c_{k \sigma}^{\dagger}$ ( $\vec{k}$ is planar momentum) 'attaches' to the string via the addition of a corner as indicated in Fig. 4 , and this particular process can be expressed by

$$
\begin{aligned}
c_{\vec{k} \uparrow}\left|\Phi_{0}\right\rangle= & \frac{1}{\sqrt{N}} \sum_{\{\eta\} \vec{k}} \alpha(\{\eta\}) e^{i\left[k_{x} \eta_{l}^{x}+k_{y}\left(\eta_{l}^{y}+1\right)\right]} a_{\eta_{l}^{x}, \eta_{l}^{y}+1}^{\dagger} \hat{A}_{l+1} \\
& \delta\left(\eta_{l+1}^{x}-\eta_{l}^{x}-1\right) \delta\left(\eta_{l+1}^{y}-\eta_{l}^{y}-1\right)|\{\eta\}\rangle .
\end{aligned}
$$

The extra hole is incorporated by deleting a diagonal link (delta functions) and replacing this by a 'corner' formed by a pair of neighbouring horizontal and vertical links. The operator $\hat{A}$ takes care of the fermion sign, and elsewhere we will discuss how the spin of the electron is linked to the orientation of the staggered order parameter in the domain from which the electron 
is removed. ${ }^{8}$ From Eq. (3) together with Eq.'s $(1,2)$, the photoemission spectrum can be calculated. The inverse photoemission follows from the conjugate of Eq. (3): adding an electron corresponds to the removal of a corner.

Let us first consider the saturated strings. Because of the dynamics, these strings are for realistic parameters (positive curvature energies) directed and the density of corners has to vanish because otherwise the curvature they induce would destroy the directedness. Since the presence of corners is associated with the presence of unoccupied elecron states, it follows that directed saturated string vacua correspond with filled band systems. It is still possible to add holes, and there is an interesting relationship between the nature of these hole states and the overall geometric properties of the string vacuum. The states directed along the $(1,0)$ direction can be thought of as a horizontal classical string, seeded with $(1, \pm 1)$ kinks. The external hole can only attach to these kinks and this results in a step-like kink, as indicated in Fig. 4: the total number of occupied electron states is given by the number of diagonal kinks. In addition, the final state kink cannot delocalize by nearest-neighbour hops: strings directed along the $(1,0)$ direction carry a relatively small number of localized electron states. This changes drastically when the string is directed along the diagonal, the fully stretched case. A hole can be added at every link, causing a corner (Fig. 4, see also Fig. 1). By single hole hops, this corner decays in a pair of freely propagating $(1,0)$ and $(0,1)$ kinks which carry both half of the hole. As will be discussed elsewhere in more detail, the one-hole spectral function corresponds with the convolution of the kink spectral functions, subjected to kinematical constraints, in analogy with the spectral functions found for $1+1 \mathrm{D}$ interacting electron systems.

It turns out that the above simple arguments hold as well in the more complicated string vacua: (under)saturated strings do not carry unoccupied electronic states and the kinks responsible for the delocalization of the holes only propagate well in fully stretched strings. This variety of strings are therefore electronic insulators, although the strings themselves might still be delocalized in space. This changes drastically when the strings are oversaturated (Fig. 3). Of particular interest is the case where the parameters are choosen such that the saturated string would orient itself along the diagonal. Forcing in more holes leads to a proliferation of the charged kinks $((1,0),(0,1)$ links) in the string ground state. Because these kinks can only propagate freely when the string is directed along the diagonal, the $(1,1)$ directed string phase is further stabilized in the oversaturated cases. One way to visualize the string vacuum is by drawing string configurations as lines with a width proportional to the probability amplitudes $|\alpha(\{\eta\})|^{2}$, accumu- 


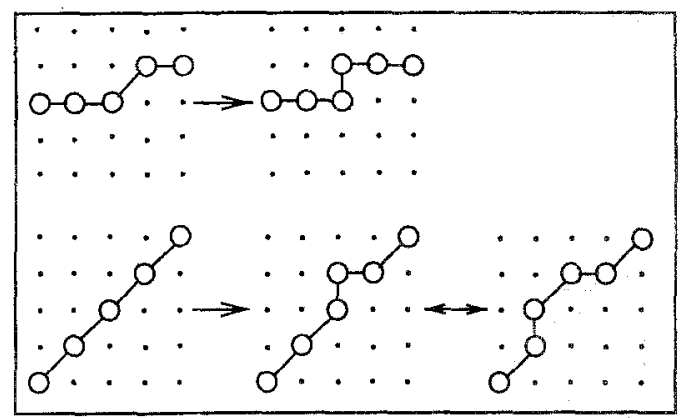

Fig. 4. A string directed in the $(1,0)$ direction is characterized by a finite density of diagonal kinks, disordering the $(1,0)$ classical string. External holes can only attach to these kinks and the resulting corner defect cannot delocalize by nearest-neighbour hops (top). If the string is directed along a diagonal direction, however, the corner caused by the external hole decays into two freely propagating kink excitations (bottom).

lating the contributions of the different string configurations on the links on the lattice. In Fig. 5 we show this for a string on a $6 \times 6$ lattice, oversaturated with 1 hole. Although the string can cover the whole plane, it quite clearly breaks the symmetry to orient itself along the diagonal direction.

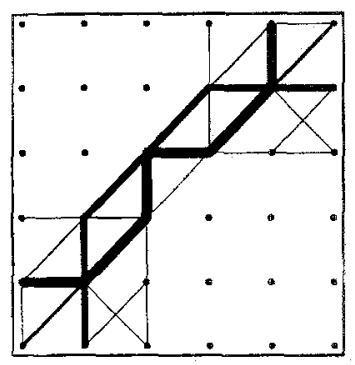

Fig. 5. Link representation of the vacuum of a $(1,1)$ directed string on a $6 \times 6$ lattice, oversaturated with one hole $\left(t=1, K=4, L_{11}=-0.8, L_{12}=\right.$ $\left.-3, L_{22}=-2\right)$.

As long as the string is directed along the $(1,1)$ direction, the charged kinks $((1,0) \sim h,(0,1) \sim v$ links $)$ themselves form a simple $1+1 \mathrm{D}$ system: (i) with respect to the $(1,1)$ 'vacuum', both types of kinks can propagate freely, (ii) the kinks cannot pass each other, and they can be considered as 
hard-core particles carrying two flavours ( $h$ and $v$ ), subject to short range repulsive interactions (the curvature energies. Obviously, the charge compressibility becomes finite in such a system, and at least the $(1,1)$ directed oversaturated string is a one dimensional metal. In addition, a large Fermisurface is expected to open up at a finite charged kink density. In order to investigate these matters in further detail, we calculated the single-electron spectral function numerically. In Fig. 6 we show the result which comes closest to a Fermi-liquid spectral function. A rather sharp peak is seen, which disperses as function of momentum, to cross the Fermi-energy at momenta roughly halfway the Brillioun zone both in the $(1,1)$ and $(1,0)$ directions, spanning up a large open Fermi-surface.

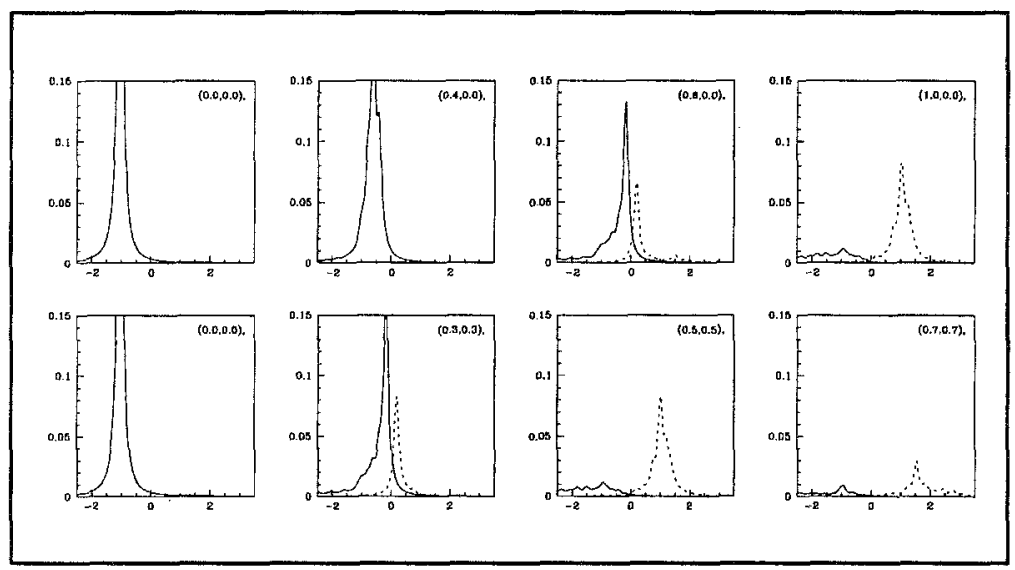

Fig. 6. Occupied- (full lines) and unoccupied part (dashed lines) of the single electron spectral function, as function of momentum in units of $\pi$ along $(1,0)$ (top) and $(1,1)$ (bottom) directions in the Brillouin zone, for a $(1,1)$ directed string on a $12 \times 12$ lattice saturated with four holes $(t=$ $\left.1, K=0, L_{11}=-1, L_{12}=0, L_{22}=0\right)$.

We assumed in the calculation an ordering of the kink flavours $h-v-$ $h-v-\cdots$, which would actually correspond with a polarization of the spins of the excess holes in the stripe interpretation. An unpolarized stripe would correspond with an ordering $h-h-v-v-\cdots$. We have also calculated the one electron spectral function for such a string, finding much the same behaviour as in Fig. (6), although the peaks are broader. Lowering the hole density has a similar effect. The width of the peaks increases, especially at larger momenta, but even at the smallest hole densities we find a sudden appearence of unoccupied states at momenta corresponding with the Fermi-surface of 
the non-interacting system. Elsewhere we will analyze the nature of the single particle spectral function of oversaturated strings in more detail. ${ }^{8}$

\section{Conclusions.}

The model described in the above is most likely a gross oversimplification of physical reality. Nevertheless, it might be that it catches some generic features of quantized stripes. (a) Microscopically, the fluctuations of the stripes are driven by the fast motions of kinks, with an associated energy scale which might well be $O(0.1) \mathrm{eV}$. The overall motion of the stripe is the result of the concerted motions of the kinks and this leads to a slow down: the string as a whole fluctuates very slowly compared to the motions of kinks. (b) For the same reasons, the stripe has a tendency to order. Although strings exists which are nominally delocalized, there is a very strong tendency for the string to take a particular direction in space. The string corresponds with a surface in Euclidean space-time, and it is well known that surfaces roughen without a proliferation of overhangs. (c) In order to remove holes locally from stripes, extreme local curvature is needed which on its turn would destroy the directedness: directed strings do not carry unoccupied electron states and are therefore electronic insulators. This changes radically when the string is forced to carry more length than is needed to span the lattice: the oversaturated string which is found after 'doping' the stripe with excess holes. Away from the saturation point, the string does not posses a preferred length and the charge compressibility becomes finite: doped stripes are metals. Since the charge is confined to move on a $1 D$ trajectory it is not surprising that such a stripe shows the generic features of a $1+1 \mathrm{D}$ metal, as a large Fermi-surface already at a low doping concentration.

It is interesting to ask if anything of this can be tested in the context of high- $T_{c}$ superconductivity. Isolated strings have a special stability at the saturation point, and the doping mechanism has to be related to the physics of the dense string liquid. As pointed out by Löw et al, ${ }^{12}$ relatively weak long range repulsive interactions will tend to cause an inter-stripe commensurability: the stripes want to keep a fixed distance. According to very recent experiments this seems to be the case both in the static striped, phase, as in the superconductors itself. ${ }^{13}$ The incommensurability of the spin peaks seen in the neutron scattering grows roughly proportional to $\mathrm{x}$ for $x=1 / 8$, to level off for larger $x$. Hence, it seems that at $x=1 / 8$ the inter-stripe commensurability takes over from the intra-stripe commensurability (the saturated strings). For $x>1 / 8$ there are two possibilities: either the excess holes become free, as proposed by Salkola et al, ${ }^{14}$ or the holes are incorporated in the strings according to the above strong-coupling perspective. It should be 
possible to discriminate between these possibilities. For instance, in strong coupling one expects quasi-one dimensional characteristics: the carriers are in first instance confined to move along lines, but the lines themselves (the strings) are delocalized in space.

\section{ACKNOWLEDGMENTS}

JZ acknowledges support by the Dutch Royal Academy of Sciences, and $\mathrm{HE}$ is supported by the Stichting voor Fundamenteel Onderzoek der Materie (FOM) which is financially supported by the Nederlandse Organisatie voor Wetenschappelijk Onderzoek (NWO).

\section{REFERENCES}

1. V. J. Emery and S. Kivelson, Phys. Rev. Lett. 74, 3253 (1995); V. J. Emery and S. Kivelson, in: The Los Alamos Symposium 1993, ed. K. S. Bedell (AddisonWesley, New York, 1994).

2. J. Zaanen and O. Gunnarson, Phys. Rev. B 40, 7391 (1989); J. Zaanen and P. B. Littlewood, Phys. Rev. B 50, 7222 (1994); J. Zaanen and A. M. Oleś, Ann. Physik 5, 224 (1996).

3. J. M. Tranquada, D. J. Buttrey, V. Sachan, and J. E. Lorenzo, Phys. Rev. Lett. 73, 1003 (1994); W. Bao, S. A. Carter, C. H. Chen, S.-W. Cheong, B. Batlogg, and Z. Fisk, preprint (AT\&T Bell Labs., 1995).

4. J. M. Tranquada, B. J. Sternlieb, J. D. Axe, Y. Nakamura, and S. Uchida, Nature 375561 (1995).

5. Similar ideas are found in C. Nayak and F. Wilczek, preprint (condmat/9602112).

6. J. Zaanen, M. Horbach, and W. van Saarloos, Phys. Rev. B 53, 8671 (1996).

7. H. Eskes, R. Grimberg, J. Zaanen and W. van Saarloos, preprint (condmat/9510129).

8. O. Y. Osman, H. Eskes, W. van Saarloos and J. Zaanen, in preparation.

9. M. den Nijs and K. Rommelse, Phys. Rev. B 40, 4709 (1989).

10. H. E. Viertiö and T. M. Rice, J. Phys.: Cond. matt. 6, 7091 (1994).

11. The director order is generally unstable to thermal fiuctuations, as expected from the Mermin-Wagner theorem.

12. U. Löw, V. J. Emery, K. Fabricius and S. A. Kivelson, Phys. Rev. Lett. 73, 1918 (1994).

13. Y. Endoh et al., unpublished; J. M. Tranquada et al., unpublished.

14. M. I. Salkola, V. J. Emery and S. A. Kivelson, preprint (cond-mat/9602102). 